Mathematical Sciences And Applications E-Notes

Volume 3 No. 1 PP. 94-102 (2015) @ MSAEN

\title{
ON $C$-TOTALLY REAL SUBMANIFOLDS OF SASAKIAN SPACE FORMS
}

\author{
AHMET YILDIZ
}

(Communicated by Bayram ȘAHIN)

\begin{abstract}
Let $\tilde{M}^{2 n+1}(c)$ be $(2 n+1)$-dimensional Sasakian space form of constant $\varphi$-sectional curvature $c$ and $M^{n}$ be an $n$-dimensional $C$-totally real minimal submanifold of $\tilde{M}^{2 n+1}(c)$. If $M^{n}$ is semi-parallel and the sectional curvature of $M^{n}$ is greater than $\frac{(n-2)(c+3)}{4(2 n-1)}$, then $M^{n}$ is totally geodesic. Then we prove that a $C$-totally real minimal surface of a 5 -dimensional Sasakian manifold $\tilde{M}(c)$ with constant $\varphi$-sectional curvature $c$, if $M$ is semi-parallel surface, then $M$ is totally geodesic or flat.
\end{abstract}

\section{Introduction}

In 1976, S. Yamaguchi, M. Kon and T. Ikawa [17] introduced the notion of a $C$-totally real submanifold of a Sasakian manifold and proved the following:

Theorem 1.1. Let $M^{2 n+1}(c)$ be a $(2 n+1)$-dimensional Sasakian manifold with constant $\varphi$-sectional curvature $c$ and $M^{n}$ be an $n$-dimensional compact $C$-totally real minimal submanifold of $\tilde{M}^{2 n+1}(c)$. If $\|\sigma\|^{2}<\frac{n(n+1)(c+3)}{4(2 n-1)}$ or, equivalently $\kappa>$ $\frac{n^{2}(n-2)(c+3)}{2(2 n-1)}$, then $M$ is totally geodesic, where $\kappa$ is the scalar curvature.

Then S. Yamaguchi, M. Kon and Y. Miyahara [16] studied a $C$-totally real minimal surface of a 5 -dimensional Sasakian manifold $\tilde{M}(c)$ with constant $\varphi$-sectional curvature $c$. They showed that if $M$ is a complete nonnegative curved surface, then $M$ is totally geodesic or flat. Then, A. Yildiz et al. [18] studied $C$-totally real pseudo-parallel submanifolds in Sasakian space forms.

Motivated by these results, in this paper we get the followings:

Theorem 1.2. Let $\tilde{M}^{2 n+1}(c)$ be a $(2 n+1)$-dimensional Sasakian space form of constant $\varphi$-sectional curvature $c$ and $M^{n}$ be an n-dimensional $C$-totally real minimal submanifold of $\tilde{M}^{2 n+1}(c)$. If $M^{n}$ is semi-parallel and the sectional curvature of $M^{n}$ is greater than $\frac{(n-2)(c+3)}{4(2 n-1)}$, then $M^{n}$ is totally geodesic.

Date: Received: January 1, 2014, Accepted: April 10, 2014.

2010 Mathematics Subject Classification. Primary 53B20, 53B25, 53B50; Secondary 53C15.

Key words and phrases. Sasakian manifolds, semi-parallel submanifolds, $C$-totally real submanifold, second fundamental form. 
Theorem 1.3. Let $M$ be a $C$-totally real minimal surface of a 5-dimensional Sasakian manifold $\tilde{M}(c)$ with constant $\varphi$-sectional curvature c. If $M$ is semi-parallel surface, then $M$ is totally geodesic or flat.

\section{Preliminaries}

Let $f: M^{n} \longrightarrow \tilde{M}^{2 n+1}(c)$ be an isometric immersion of an $n$-dimensional Riemannian manifold $M$ into $(2 n+1)$-dimensional space form $\tilde{M}^{2 n+1}(c)$. We denote by $\nabla$ and $\tilde{\nabla}$ the Levi-Civita connections of $M$ and $\tilde{M}^{2 n+1}(c)$ respectively, and $N(M)$ its normal bundle. Then for vector fields $X, Y$ which are tangent to $M$, the second fundamental form $\sigma$ is given by the formula $\sigma(X, Y)=\tilde{\nabla}_{X} Y-\nabla_{X} Y$. Furthermore, for $\xi \in N(M), A_{\xi}: T M \longrightarrow T M$ will denote the Weingarten operator in the direction $\xi, A_{\xi} X=\nabla_{X}^{\perp} \xi-\tilde{\nabla}_{X} \xi$, where $\nabla^{\perp}$ denotes the normal connection of $M$. The second fundamental form $\sigma$ and $A_{\xi}$ are related by $\tilde{g}(\sigma(X, Y), \xi)=g\left(A_{\xi} X, Y\right)$, where $g$ is the induced metric of $\tilde{g}$ for any vector fields $X, Y$ tangent to $M$. The mean curvature vector $H$ of $M$ is defined to be

$$
H=\frac{1}{n} \operatorname{Tr}(\sigma) .
$$

A submanifold $M$ is said to be a minimal submanifold in $\tilde{M}$ if its mean curvature vector $H$ is identically zero. Moreover, $M$ is called a totally geodesic submanifold in $\tilde{M}$ if its second fundamental form $\sigma$ is identically zero. The covariant derivative $\bar{\nabla} \sigma$ of $\sigma$ is defined by

$$
\left(\bar{\nabla}_{X} \sigma\right)(Y, Z)=\nabla_{X}^{\perp}(\sigma(Y, Z))-\sigma\left(\nabla_{X} Y, Z\right)-\sigma\left(Y, \nabla_{X} Z\right),
$$

where, $\bar{\nabla} \sigma$ is a normal bundle valued tensor of type $(0,3)$ and is called the third fundamental form of $M$. The equation of Codazzi implies that $\bar{\nabla} \sigma$ is symmetric hence

$$
\left(\bar{\nabla}_{X} \sigma\right)(Y, Z)=\left(\bar{\nabla}_{Y} \sigma\right)(X, Z)=\left(\bar{\nabla}_{Z} \sigma\right)(X, Y) .
$$

Here, $\bar{\nabla}$ is called the van der Waerden-Bortolotti connection $\bar{\nabla}=\nabla \oplus \nabla^{\perp}$, where $\nabla$ is the Levi-Civita connection and $\nabla^{\perp}$ is the normal connection of $M^{n}$. Given an isometric immersion $f: M \longrightarrow \tilde{M}$, if $\bar{\nabla} \sigma=0$, then $f$ is called parallel [10]. Then J. Deprez ([7], [8]) defined the immersion to be semi-parallel if

$$
\bar{R}(X, Y) \cdot \sigma=0 .
$$

The basic equations of Gauss and Ricci are defined by

$$
\begin{gathered}
g(R(X, Y) Z, W)=\frac{c+3}{4}(g(Y, Z) g(X, W)-g(X, Z) g(Y, W)) \\
\quad+\sum_{\alpha}\left(g\left(A_{\alpha} X, W\right)\left(g\left(A_{\alpha} Y, Z\right)-g\left(A_{\alpha} X, Z\right) g\left(A_{\alpha} Y, W\right)\right),\right. \\
g\left(R^{\perp}(X, Y) \xi, \eta\right)=g\left(\left[A_{\xi}, A_{\eta}\right] X, Y\right) ; \quad \xi, \eta \in N(M),
\end{gathered}
$$

respectively. Where $R^{\perp}$ is the curvature operator of the normal connection defined by

$$
R^{\perp}(X, Y) Z=\nabla_{X}^{\perp} \nabla_{Y}^{\perp} Z-\nabla_{Y}^{\perp} \nabla_{X}^{\perp} Z-\nabla_{[X, Y]}^{\perp} Z .
$$

An isometric immersion $f$ (or the submanifold $M$ ) is said to have flat normal connection (or trivial normal connection) if $R^{\perp}=0$. If $M$ has flat normal connection then shortly we call it to be normally flat. The relation (2.4) shows that the triviality of the normal connection of $M$ into space form $\mathbb{N}^{n+d}(c)$ (and more generally, for 
submanifolds in a locally conformally flat space) is equivalent to the fact that all second fundamental tensors are mutually commute, or that all second fundamental tensors are mutually diagonalizable [5].

The sectional curvature $K(X, Y)$ of $M$ determined by an orthonormal pair $X, Y$ is given by

$$
K(X, Y)=\frac{c+3}{4}+\sum_{\alpha}\left(g\left(A_{\alpha} X, X\right) g\left(A_{\alpha} Y, Y\right)-g\left(A_{\alpha} X, Y\right)^{2}\right) .
$$

The second covariant derivative $\bar{\nabla}^{2} \sigma$ of $\sigma$ is defined by

$$
\begin{aligned}
\left(\bar{\nabla}^{2} \sigma\right)(Z, W, X, Y)= & \left(\bar{\nabla}_{X} \bar{\nabla}_{Y} \sigma\right)(Z, W) \\
= & \nabla_{X}^{\perp}\left(\left(\bar{\nabla}_{Y} \sigma\right)(Z, W)\right)-\left(\bar{\nabla}_{Y} \sigma\right)\left(\nabla_{X} Z, W\right) \\
& -\left(\bar{\nabla}_{X} \sigma\right)\left(Z, \nabla_{Y} W\right)-\left(\bar{\nabla}_{\nabla_{X} Y} \sigma\right)(Z, W) .
\end{aligned}
$$

Then we have

$$
\begin{aligned}
\left(\bar{\nabla}_{X} \bar{\nabla}_{Y} \sigma\right)(Z, W)-\left(\bar{\nabla}_{Y} \bar{\nabla}_{X} \sigma\right)(Z, W)= & (\bar{R}(X, Y) \cdot \sigma)(Z, W) \\
(2.6)= & R^{\perp}(X, Y) \sigma(Z, W)-\sigma(R(X, Y) Z, W) \\
& -\sigma(Z, R(X, Y) W) .
\end{aligned}
$$

where $\bar{R}$ is the curvature tensor belonging to the connection $\bar{\nabla}$.

\section{C-totally real submanifolds of Sasakian space forms}

Let $\tilde{M}$ be a $(2 n+1)$-dimensional manifold and $\Gamma(\tilde{M})$ be the Lie algebra of vector fields on $\tilde{M}$. An almost contact structure on $\tilde{M}$ is defined by a $(1,1)$-tensor $\varphi$, a vector field $\xi$ and a 1-form $\eta$ on $\tilde{M}$ satisfy

$$
\varphi^{2} X=-X+\eta(X) \xi, \quad \varphi \xi=0, \quad \eta(\varphi X)=0, \quad \eta(\xi)=1,
$$

where $X \in \Gamma(\tilde{M})$.Manifolds equipped with an almost contact structure are called almost contact manifolds. A Riemannian manifold $\tilde{M}$ with metric tensor $g$ and almost contact structure $(\varphi, \xi, \eta)$ such that

$$
g(\varphi X, \varphi Y)=g(X, Y)-\eta(X) \eta(Y),
$$

or equivalently

$$
g(X, \varphi Y)=-g(\varphi X, Y) \text { and } \eta(X)=g(X, \xi),
$$

for all $X, Y \in \Gamma(\tilde{M})$, is almost contact metric manifold. The existence of an almost contact metric structure on $\tilde{M}$ is equivalent with the existence of a reduction of the structurel group to $U(n) \times 1$, i.e., all the matrices of $O(2 n+1)$ of the form

$$
\left(\begin{array}{ccc}
A & B & 0 \\
-B & A & 0 \\
0 & 0 & 1
\end{array}\right)
$$

where $A$ and $B$ are real $(n \times n)$-matrices. The fundamental 2-form $\Psi$ of an almost contact metric manifold $(\tilde{M}, \varphi, \xi, \eta, g)$ is defined by

$$
\Psi(X, Y)=g(\varphi X, Y)
$$


for all $X, Y \in \Gamma(\tilde{M})$, and this form satisfies $\eta \wedge \Psi^{n} \neq 0$. When $\Psi=d \eta$ the associated structure is a contact structure and $\tilde{M}$ is an almost Sasakian manifold. We denote by $\tilde{\nabla}$ the Levi-Civita connection on $\tilde{M}$. Then we have [13]

$$
\left(\tilde{\nabla}_{X} \varphi\right) Y=g(X, Y) \xi-\eta(Y) X, \quad \tilde{\nabla}_{X} \xi=-\varphi X,
$$

for any vector fields $X, Y$ tangent to $\tilde{M}$.

If moreover the structure is normal, that is, if $[\varphi X, \varphi Y]+\varphi^{2}[X, Y]-\varphi[X, \varphi Y]-$ $\varphi[\varphi X, Y]=-2 d \eta(X, Y) \xi$, then the contact metric structure is called a Sasakian structure (normal contact metric structure) and $\tilde{M}^{2 n+1}$ is called a Sasakian manifold. For more details and background, see the standard references ([2], [15].

A plane section in the tangent space $T_{X} \tilde{M}$ at $x \in \tilde{M}$ is called a $\varphi$-section if it is spanned by a vector $X$ orthogonal to $\xi$ and $\varphi X$. The sectional curvature $K(X, \varphi X)$ with respect to a $\varphi$-section determined by a vector $X$ is called a $\varphi$ sectional curvature. A Sasakian manifold with constant $\varphi$-sectional curvature $c$ is a Sasakian space form and is denoted by $\tilde{M}(c)$. The curvature tensor of a Sasakian space form $\tilde{M}(c)$ is given by

$$
\begin{aligned}
\tilde{R}(X, Y) Z= & \frac{1}{4}(c+3)\{g(Y, Z) X-g(X, Z) Y\} \\
& -\frac{1}{4}(c-1)\left\{\begin{array}{c}
\eta(Y) \eta(Z) X-\eta(X) \eta(Z) Y+g(Y, Z) \eta(X) \xi \\
-g(X, Z) \eta(Y) \xi-g(\varphi Y, Z) \varphi X \\
+g(\varphi X, Z) \varphi Y+2 g(\varphi X, Y) \varphi Z
\end{array}\right\} .
\end{aligned}
$$

Example 3.1. [2] Let $\mathbb{R}^{2 n+1}$ be a Euclidean space with cartesian coordinates $\left(x^{i}, y^{i}, z\right)$. Then a Sasakian structure on $\mathbb{R}^{2 n+1}$ is defined by $(\varphi, \xi, \eta, g)$ such that

$$
\xi=2 \frac{\partial}{\partial z}, \quad \eta=\frac{1}{2}\left(d z-\sum_{i=1}^{m} y^{i} d x^{i}\right), g=\frac{1}{4}\left[\eta \otimes \eta+\sum_{i=1}^{m}\left(\left(d x^{i}\right)^{2}+\left(d y^{i}\right)^{2}\right)\right],
$$

and the tensor field $\varphi$ is given by matrix

$$
\left(\begin{array}{ccc}
0 & \delta_{i j} & 0 \\
-\delta_{i j} & 0 & 0 \\
0 & y^{j} & 0
\end{array}\right) .
$$

With such a structure, $\mathbb{R}^{2 n+1}$ is of constant $\varphi$-sectional curvature -3 and denoted by $\mathbb{R}^{2 n+1}(-3)$.

Example 3.2. [1] For $\theta \in(0, \pi / 2)$, the immersion

$$
F(u, v, w, s, t)=2(u, 0, w, 0, v \cos \theta, v \sin \theta, s \cos \theta, s \sin \theta, t),
$$

defines a 5 -dimensional submanifold $M$ in $\mathbb{R}^{9}(-3)$. We consider on $M$ the induced almost contact structure $(\varphi, \xi, \eta, g)$, where $\varphi=(\sec \theta) T, T$ being the tangential component of $\varphi$. It can be checked that

$$
\left(\nabla_{X} \varphi\right) Y=\cos \theta(g(X, Y) \xi-\eta(Y) X),
$$

for any vector fields $X, Y$ tangent to $M$.

We remark that the immersion $F$ in the Example 2 defines a 5-dimensional minimal submanifold $M$ in a Sasakian space form $\mathbb{R}^{9}(-3)$.

A submanifold $M$ of a Sasakian manifold $\tilde{M}$ is called a $C$-totally real submanifold if and only if $\varphi\left(T_{x} M\right) \subset T_{x}^{\perp} M$ for all $x \in M$ ( $T_{x} M$ and $T_{x}^{\perp} M$ are respectively the tangent space and normal space of $M$ at $x$ ). 
When $\xi$ is tangent to $M$ (e.g., when $m=n-1$ ), $M$ is a $C$-totally real submanifold if and only if $\nabla_{X} \xi=0$ for all $X \in T M$, where $\nabla$ is the connection on $M$ induced from the Levi-Civita connection $\tilde{\nabla}$ on $\tilde{M}$. For a $C$-totally real submanifold $M^{m+1}$ in $\tilde{M}^{2(m+p)+1}$ with $m \geq 1$ it is impossible for $M$ to be totally umbilical [11].

When $\xi$ is normal to $M$ then the submanifold $M$ is automatically anti-invariant in $\tilde{M}$ with $m \leq n$ and also $\eta(X)=g(X, \xi)=0$ for $X \in T M$ [14]. On the other hand, $\eta=0$ defines a $n$-dimensional distribution on $\tilde{M}$, the so called contact distribution $D$. This distribution admits integral submanifolds up to (and including) dimension $n$. Moreover, it is proved that a manifold $M$ immersed in $\tilde{M}$ is an integral submanifold of $D$ if and only if $T_{x} M \subset D_{x}$ for $x \in M$ and $\varphi\left(T_{x} M\right) \subset T_{x}^{\perp} M$ [3]. The integral submanifols of the contact distribution of a Sasakian manifold are called $C$-totally real submanifolds. It is easy to see that the $C$-totally real submanifolds $M$ of $\tilde{M}$ are the submanifolds with $\xi \in T^{\perp} M$. [12]:

Then we have known $\operatorname{dim} M \leq n$ and the following theorem has been proved

Theorem 3.1. Let $M$ be an $m(m \leq n)$ dimensional $C$-totally real submanifold in a Sasakian manifold $\tilde{M}^{2 n+1}$ with structure tensors $(\varphi, \xi, \eta, g)$. Then we have the following:

(i): The second fundamental form of $\xi$ direction is identically zero.

(ii): If $X \in \chi(M)$, then $\varphi X \in \chi^{\perp}(M)$.

(iii): If $m=n$, then $A_{\varphi X}(Y)=A_{\varphi Y}(X), X, Y \in \chi(M)$.

Also, we need the followings:

Lemma 3.1. [15] Let $M$ be an $n$-dimensional $C$-totally real submanifold of a $(2 n+1)$-dimensional Sasakian manifold $\tilde{M}$. If the second fundamental form of $M$ is parallel, then $M$ is totally geodesic in $\tilde{M}$.

Lemma 3.2. [2] If the sectional curvature of $M^{n}$ is greater than $\delta$, then

$$
\begin{aligned}
\frac{1}{2} \Delta\left(\|\sigma\|^{2}\right) \geq & \|\bar{\nabla} \sigma\|^{2}+(1+a) n \delta\|\sigma\|^{2}-\frac{n a(c+3)-(c-1)}{4}\|\sigma\|^{2} \\
& +\frac{1-a}{2} \sum_{\alpha, \beta} \operatorname{tr}\left(A_{\alpha} A_{\beta}-A_{\beta} A_{\alpha}\right)^{2}+a \sum_{\alpha, \beta} \operatorname{tr}\left(A_{\alpha} A_{\beta}\right)^{2}
\end{aligned}
$$

for any real number $a \geq-1$.

Lemma 3.3. $[2]\|\bar{\nabla} \sigma\|^{2} \geq\|\sigma\|^{2}$.

Proposition 3.1. [9] If $M$ is an $n$-dimensional $C$-totally real submanifold of a Sasakian space form $\tilde{M}(c)$, then the following conditions are equivalent: $(i) M$ is minimal; (ii) the mean curvature vector $H$ of $M$ is parallel.

\section{Proofs of the Theorems}

Let $M$ be an $n$-dimensional $C$-totally real submanifold of $(2 n+1)$-dimensional Sasakian space form $\tilde{M}^{2 n+1}(c)$ of constant $\varphi$-sectional curvature $c$. We choose an orthonormal bases $\left\{e_{1}, e_{2}, \ldots, e_{n}, \varphi e_{1}=e_{1^{*}}, \ldots, \varphi e_{n}=e_{n^{*}}, e_{(n+1)^{*}}=\xi\right\}$. Then for $1 \leq i, j \leq n, n+1 \leq \alpha \leq 2 n+1$, the components of the second fundamental form $\sigma$ are given by

$$
\sigma_{i j}^{\alpha}=g\left(\sigma\left(e_{i}, e_{j}\right), e_{\alpha}\right)
$$


Similarly, the components of the first and the second covariant derivative of $\sigma$ are given by

$$
\sigma_{i j k}^{\alpha}=g\left(\left(\bar{\nabla}_{e_{k}} \sigma\right)\left(e_{i}, e_{j}\right), e_{\alpha}\right)=\bar{\nabla}_{e_{k}}^{\alpha} \sigma_{i j}
$$

and

$$
\begin{aligned}
\sigma_{i j k l}^{\alpha} & =g\left(\left(\bar{\nabla}_{e_{l}} \bar{\nabla}_{e_{k}} \sigma\right)\left(e_{i}, e_{j}\right), e_{\alpha}\right) \\
& =\bar{\nabla}_{e_{l}}^{\alpha} \sigma_{i j k} \\
& =\bar{\nabla}_{e_{l}} \bar{\nabla}_{e_{k}}^{\alpha} \sigma_{i j},
\end{aligned}
$$

respectively. If $f$ is semi-parallel, then by definition, the condition

$$
\bar{R}\left(e_{l}, e_{k}\right) \cdot \sigma=0 \text {. }
$$

By (2.6), we have

$$
\left(\bar{R}\left(e_{l}, e_{k}\right) \cdot \sigma\right)\left(e_{i}, e_{j}\right)=\left(\bar{\nabla}_{e_{l}} \bar{\nabla}_{e_{k}} \sigma\right)\left(e_{i}, e_{j}\right)-\left(\bar{\nabla}_{e_{k}} \bar{\nabla}_{e_{l}} \sigma\right)\left(e_{i}, e_{j}\right)
$$

Making use of (4.1), (4.3), (4.5), the semi-parallelity condition (4.4) turns into

$$
\sigma_{i j k l}^{\alpha}-\sigma_{i j l k}^{\alpha}=0 \text {. }
$$

Recall that the Laplacian $\Delta \sigma_{i j}^{\alpha}$ of $\sigma_{i j}^{\alpha}$ is defined by

$$
\Delta \sigma_{i j}^{\alpha}=\sum_{i, j, k=1}^{n} \sigma_{i j k k}^{\alpha}
$$

Then we obtain

$$
\frac{1}{2} \Delta\left(\|\sigma\|^{2}\right)=\sum_{i, j, k=1}^{n} \sum_{\alpha=1}^{p} \sigma_{i j}^{\alpha} \sigma_{i j k k}^{\alpha}+\|\bar{\nabla} \sigma\|^{2}
$$

where

$$
\|\sigma\|^{2}=\sum_{i, j, k=1}^{n} \sum_{\alpha=1}^{p}\left(\sigma_{i j}^{\alpha}\right)^{2}
$$

and

$$
\|\bar{\nabla} \sigma\|^{2}=\sum_{i, j, k=1}^{n} \sum_{\alpha=1}^{p}\left(\sigma_{i j k k}^{\alpha}\right)^{2},
$$

are the square of the length of the second and the third fundamental forms of $M^{n}$, respectively. In addition, making use of (4.1) and (4.3), we obtain

$$
\begin{aligned}
\sigma_{i j}^{\alpha} \sigma_{i j k k}^{\alpha} & =g\left(\sigma\left(e_{i}, e_{j}\right), e_{\alpha}\right) g\left(\left(\bar{\nabla}_{e_{k}} \bar{\nabla}_{e_{k}} \sigma\right)\left(e_{i}, e_{j}\right), e_{\alpha}\right) \\
& =g\left(\left(\bar{\nabla}_{e_{k}} \bar{\nabla}_{e_{k}} \sigma\right)\left(e_{i}, e_{j}\right) g\left(\sigma\left(e_{i}, e_{j}\right), e_{\alpha}\right), e_{\alpha}\right) \\
& =g\left(\left(\bar{\nabla}_{e_{k}} \bar{\nabla}_{e_{k}} \sigma\right)\left(e_{i}, e_{j}\right), \sigma\left(e_{i}, e_{j}\right)\right) .
\end{aligned}
$$

Due to (4.11), the equation (4.8) becomes

$$
\frac{1}{2} \Delta\left(\|\sigma\|^{2}\right)=\sum_{i, j, k=1}^{n} g\left(\left(\bar{\nabla}_{e_{k}} \bar{\nabla}_{e_{k}} \sigma\right)\left(e_{i}, e_{j}\right), \sigma\left(e_{i}, e_{j}\right)\right)+\|\bar{\nabla} \sigma\|^{2} .
$$

Further, by the use of (4.4) and (4.5), we get

$$
\begin{aligned}
g\left(\left(\bar{\nabla}_{e_{k}} \bar{\nabla}_{e_{k}} \sigma\right)\left(e_{i}, e_{j}\right), \sigma\left(e_{i}, e_{j}\right)\right. & =g\left(\left(\bar{\nabla}_{e_{k}} \bar{\nabla}_{e_{i}} \sigma\right)\left(e_{k}, e_{j}\right), \sigma\left(e_{i}, e_{j}\right)\right) \\
& =g\left(\left(\bar{\nabla}_{e_{i}} \bar{\nabla}_{e_{k}} \sigma\right)\left(e_{j}, e_{k}\right), \sigma\left(e_{i}, e_{j}\right)\right)
\end{aligned}
$$


Substituting (4.13) into (4.12), we have

$$
\begin{aligned}
\frac{1}{2} \Delta\left(\|\sigma\|^{2}\right)= & \sum_{i, j, k=1}^{n}\left[g\left(\left(\bar{\nabla}_{e_{i}} \bar{\nabla}_{e_{j}} \sigma\right)\left(e_{k}, e_{k}\right), \sigma\left(e_{i}, e_{j}\right)\right)\right. \\
& +\|\bar{\nabla} \sigma\|^{2}
\end{aligned}
$$

Furthermore, by the definition

$$
\begin{gathered}
\|\sigma\|^{2}=\sum_{i, j=1}^{n} g\left(\sigma\left(e_{i}, e_{j}\right), \sigma\left(e_{i}, e_{j}\right)\right), \\
H^{\alpha}=\sum_{k=1}^{n} \sigma_{k k}^{\alpha} \\
\|H\|^{2}=\frac{1}{n^{2}} \sum_{\alpha=1}^{p}\left(H^{\alpha}\right)^{2} .
\end{gathered}
$$

After some calculations, we get

$$
\begin{aligned}
\frac{1}{2} \Delta\left(\|\sigma\|^{2}\right)= & \sum_{i, j=1}^{n} \sum_{\alpha=1}^{p} \sigma_{i j}^{\alpha}\left(\bar{\nabla}_{e_{i}} \bar{\nabla}_{e_{j}} H^{\alpha}\right) \\
& +\|\bar{\nabla} \sigma\|^{2} .
\end{aligned}
$$

Using minimallity condition, the equation (4.18) reduces to

$$
\frac{1}{2} \Delta\left(\|\sigma\|^{2}\right)=\|\bar{\nabla} \sigma\|^{2} .
$$

On the other hand Blair [2] shown that

$$
\begin{aligned}
\frac{1}{2} \Delta\left(\|\sigma\|^{2}\right) \geq & \|\bar{\nabla} \sigma\|^{2}+(1+a) n \delta\|\sigma\|^{2}-\frac{(n a-1)(c+3)}{4}\|\sigma\|^{2} \\
& -(1-a)\|\sigma\|^{4}+\sum_{i=1}^{n}\left(\operatorname{tr} A_{i}^{2}\right)^{2}
\end{aligned}
$$

for $-1 \leq a \leq 1$. Hence using (4.19) in (4.20), we have

$$
\begin{aligned}
0 \geqslant & (1+a) n \delta\|\sigma\|^{2}-\frac{(n a-1)(c+3)}{4}\|\sigma\|^{2} \\
& -(1-a)\|\sigma\|^{4}+\sum_{i=1}^{n}\left(\operatorname{tr} A_{i}^{2}\right)^{2}
\end{aligned}
$$

for $-1 \leq a \leq 1$. Moreover one can easily show that

$$
\sum_{\alpha, \beta=1}^{n}\left(\operatorname{tr} A_{\alpha} A_{\beta}\right)^{2} \geqslant \frac{1}{n}\|\sigma\|^{4} .
$$

Thus we have

$$
\begin{aligned}
0 \geqslant & (1+a) n \delta\|\sigma\|^{2}-\frac{(n a-1)(c+3)}{4}\|\sigma\|^{2} \\
& +\left(\frac{1}{n}-(1-a)\right)\|\sigma\|^{4} .
\end{aligned}
$$


Setting $a=1-\frac{1}{n}$ in $(4.21)$, we obtain

$$
0 \geqslant\left[(2 n-1) \delta-\frac{(n-2)(c+3)}{4}\right]\|\sigma\|^{2} .
$$

If $\delta>\frac{(n-2)(c+3)}{4(2 n-1)}$ then $\|\sigma\|^{2}=0$, i.e. $\sigma=0$. This completes the proof of Theorem 2 .

Now we assume that $\tilde{M}(c)$ is a 5 -dimensional Sasakian manifold with constant $\varphi$-sectional curvature $c$ and $M$ is a $C$-totally real minimal surface of $\tilde{M}(c)$. Now we take a frame $e_{1}, e_{2}$ for $T_{p}(M)$ and a frame $\varphi e_{1}, \varphi e_{2}, \xi$ for $T_{p}(M)^{\perp}$. Then the second fundamental form can be expressed as:

$$
A_{\varphi e_{1}}=\left(\begin{array}{cc}
b & 0 \\
0 & -b
\end{array}\right), \quad A_{\varphi e_{2}}=\left(\begin{array}{cc}
0 & -b \\
-b & 0
\end{array}\right), \quad A_{\xi}=\left(\begin{array}{ll}
0 & 0 \\
0 & 0
\end{array}\right) .
$$

From (2.3) and (2.4), we obtain

$$
\begin{aligned}
R\left(e_{1}, e_{2}\right) e_{1} & =\left(-\frac{c+3}{4}+2 b^{2}\right) e_{2}, \\
R\left(e_{1}, e_{2}\right) e_{2} & =\left(\frac{c+3}{4}-2 b^{2}\right) e_{1}, \\
R^{\perp}\left(e_{1}, e_{2}\right) \varphi e_{1} & =2 b^{2} \varphi e_{2}, \\
R^{\perp}\left(e_{1}, e_{2}\right) \varphi e_{2} & =-2 b^{2} \varphi e_{1} .
\end{aligned}
$$

Moreover, by the Gauss equation, we have

$$
2 b^{2}=\frac{c+3}{4}-\gamma,
$$

where $\gamma$ denotes the Gauss curvature of $M$. If $M$ is semi-parallel surface, then we obtain

$$
\begin{aligned}
& \left(R\left(e_{1}, e_{2}\right) \cdot \sigma\right)\left(e_{1}, e_{1}\right)=6 b^{3}-\frac{b(c+3)}{2}=0 \\
& \left(R\left(e_{1}, e_{2}\right) \cdot \sigma\right)\left(e_{2}, e_{2}\right)=-6 b^{3}+\frac{b(c+3)}{2}=0 \\
& \left(R\left(e_{1}, e_{2}\right) \cdot \sigma\right)\left(e_{1}, e_{2}\right)=6 b^{3}-\frac{b(c+3)}{2}=0
\end{aligned}
$$

which give that

$$
\left(b^{2}-\gamma\right) b=0
$$

Now we have two cases:

Case i) If $\gamma=b^{2}>0$, then from [16], we can say that $M$ is totally geodesic or, Case ii) If $b=0$, then we can say that $M$ is flat.

This completes the proof of Theorem 1.3.

\section{REFERENCES}

[1] Alegre P. and Carriazo A., Structures on generalized Sasakian-space-forms, Differential Geom. Appl., 26(2008), 656-666.

[2] Blair, D.E., Contact manifolds in Riemannian geometry, Lectures Notes in Mathematics 509, Springer-Verlag, Berlin, (1976), 146p.

[3] Blair D.E., and Ogiue, K., Geometry of Integral submanifolds of a Contact Distribution, Illinois J.Math., 19(1975), 269-27.

[4] Boeckx, E., Kowalski, O. and Vanhecke, L., Riemannian Manifolds of Conullity Two, World Sc., Singapore, (1996).

[5] Chen, B.Y., Geometry of Submanifolds, Dekker, (1973). 
[6] Chern, S.S., M.P.do Carmo and Kobayashi, S., Minimal submanifolds of sphere with second fundamental form of constant length, Functional Analysis and Related Fields, Springer- Verlag (1970), 59-75.

[7] Deprez, J., Semi-Parallel Surfaces in Euclidean space, J. Geom., 25(1985), 192-200.

[8] Deprez, J., Semi-Parallel Hypersurfaces, Rend. Sem. Mat. Univers. Politecn. Torino, 44(1986), 303-316.

[9] Dillen, F., Vrancken, L., C-totally Real Submanifolds of Sasakian Space Forms, J. Math. Pures et Appl., 69(1990), 85-93.

[10] Ferus, D., Immersions with parallel second fundamental form, Math. Z., 140(1974), 87-93.

[11] Kon, M., Remarks on Anti-invariant Submanifolds of a Sasakian Manifold, Tensor N.S., 30(1976), 239-246.

[12] Yamaguchi S., Ikawa T., On compact minimal C-totally real submanifolds, Tensor 29(1975), 30-34.

[13] Yano K., Kon M., Anti-invariant Submanifolds of a Sasakian Space Forms, Tohoku Math.J., 29(1976), 9-23.

[14] Yano K., Kon M., Anti-invariant Submanifolds, Marcel Dekker, New York, (1978).

[15] Yano K., Kon M., Structures on manifolds, World Scientific, (1984), 508p.

[16] Yamaguchi S., Kon M. and Miyahara Y., A theorem on C-totally real minimal surface, Proceedings of the American Math. Soc., 54(1976), 276-280.

[17] Yamaguchi S., Kon M. and Ikawa T., C-totally real submanifolds, J. Differential Geometry, 11(1976), 59-64.

[18] Yıldız A., Murathan C., Arslan K. and Ezentaş R., C-totally real pseudo parallel submanifolds of Sasakian space forms, Monatshefte für Mathematik, 151, no. 3, (2007), 247-256.

Education Faculty, Department of Mathematics, Inonu University, Malatya, TURKEy

E-mail address: a.yildiz@inonu.edu.tr; ayildiz44@yahoo.com 